\title{
Evaluation of the factors that affecting quality of life of children with cystic fibrosis
}

Zeynep Kayra Tanrıverdi, Velat Sen, Hadice Selimoglu Sen, İsmail Yıldız

Dicle University School of Medicine

Department of Pediatric Pulmonology Diyarbakır/Turkey

OBJECTIVE: Cystic fibrosis (CF) is a chronic disease affecting both the child and the family system. The aim of this study was to evalute the factors that affect the quality of life of children with CF.

MATERIAL AND METHODS: The study included 40 children with CF and the mothers of 40 children with CF. The sociodemographic information and Cystic Fibrosis Questionnaire-Revised (CFQ-R;), form were used in the evaluation of the subjects.

RESULTS: The CFQ-R scores of the parents of the CF patients who were admitted to the hospital were higher than the mothers of non-hospitalized children. Additionally; there was a statistically significant difference in the treatment and nutritional scores in the between two groups. Also there was a statistically significant difference only in the physical functioning parameters between the patients who were hospitalized and non-hospitalized.

CFQ-R scores of the parents of CF patients who were diagnosed with pseudomonas aeruginosa (PA) infection were higher than the mothers of children without PA infection. There was a significant correlation in vitality, treatment, health, body image, and digestion scores.

The CF patients with BMI below 25th percentile were lower physical functioning, vitality, body image, weight, nutritional symptoms and school performance scores than the patients with BMI above 25th percentile.

CONCLUSION: Pseudomonas aeruginosa infection, hospitalization and malnutrition negatively affected the quality of life of the CF patients and mothers of children with CF. There is a need for further studies and developing programs that will provide psychosocial support for mothers of children with CF in our country.

Key Words: Cystic Fibrosis Questionnaire-Revised, Childhood, Parent, Cystic fibrosis 